\title{
Do assessments of cardiorespiratory and muscular fitness influence subsequent reported physical activity? A randomized controlled trial
}

\author{
James T. Langland ${ }^{1 *}$ (D, Neeraj Sathnur ${ }^{2}$, Qi Wang ${ }^{3}$ and Andrew P. J. Olson ${ }^{1}$
}

\begin{abstract}
Background: Regular physical activity and exercise provide many health benefits. These health benefits are mediated in large part through cardiorespiratory fitness and muscular strength. As most individuals have not had an assessment of their personal cardiorespiratory fitness or muscular strength we investigated if measurements of cardiorespiratory fitness and muscular strength would influence an individual's subsequent self-reported exercise and physical activity.

Methods: Volunteer subjects at a State Fair were randomized in 1:1 parallel fashion to control and intervention groups. The baseline Exercise Vital Sign (EVS) and type of physical activity were obtained from all subjects. The intervention group received estimated maximum oxygen uptake $\left(\mathrm{VO}_{2} \mathrm{max}\right)$ using a step test and muscular strength using a hand grip dynamometer along with age-specific norms for both measurements. All subjects were provided exercise recommendations. Follow up surveys were conducted at 3,6 and 12 months regarding their EVS and physical activity.

Results: One thousand three hundred fifteen individuals (656 intervention, 659 control) were randomized with 1 year follow up data obtained from 823 subjects (62.5\%). Baseline mean EVS was 213 min/week. No change in EVS was found in either group at follow-up $(p=0.99)$. Subjects who were less active at baseline (EVS $<150)$ did show an increase in EVS (86 to 146) at 6 months $(p<0.05)$. At 3 months the intervention group increased resistance training (29.1 to $42.8 \%$ ) compared to controls ( 26.3 to $31.4 \%$ ) $(p<0.05)$. Lifestyle physical activity increased in the intervention group at 3 months (27.7 to $29.1 \%)$ and 6 months (25\%) whereas it declined in the control group at 3 months (24.4 to $20.1 \%)$ and 6 months $(18.7 \%)(p<0.05)$.

Conclusion: Providing $\mathrm{VO}_{2}$ max estimates and grip strength did not produce an increase in overall physical activity. The EVS and exercise recommendations did however produce an increase in physical activity in less active individuals. In a very active population the $\mathrm{VO}_{2}$ max estimate and measured grip strength did increase lifestyle activity and resistance training. Wider adoption of these measures could be effective in promoting physical activity and resistance training.
\end{abstract}

\footnotetext{
*Correspondence: langl039@umn.edu

'Department of Medicine, Division of General Internal Medicine, University of Minnesota, 420 Delaware St SE, MMC 784, Minneapolis, MN 55455, USA

Full list of author information is available at the end of the article
}

(c) The Author(s). 2021 Open Access This article is licensed under a Creative Commons Attribution 4.0 International License, which permits use, sharing, adaptation, distribution and reproduction in any medium or format, as long as you give appropriate credit to the original author(s) and the source, provide a link to the Creative Commons licence, and indicate if changes were made. The images or other third party material in this article are included in the article's Creative Commons licence, unless indicated otherwise in a credit line to the material. If material is not included in the article's Creative Commons licence and your intended use is not permitted by statutory regulation or exceeds the permitted use, you will need to obtain permission directly from the copyright holder. To view a copy of this licence, visit http://creativecommons.org/licenses/by/4.0/. The Creative Commons Public Domain Dedication waiver (http://creativecommons.org/publicdomain/zero/1.0/) applies to the data made available in this article, unless otherwise stated in a credit line to the data. 
Trial registration: clinicaltrials.gov NCT03518931 Registered 05/08/2018 -retrospectively registered.

Keywords: Cardiorespiratory fitness, Grip strength, Physical activity, Exercise, Exercise promotion

\section{Background}

Physical activity and regular exercise are important components of a healthy lifestyle. The benefits of physical activity and exercise include lower all-cause mortality [1, 2], reduced cardiovascular disease (CVD) [3], improved blood pressure [4], lower triglycerides, increased High Density Lipoprotein cholesterol [5], less depression [6], less anxiety [7], improved cognitive function [8], and improved glycemic control in both type 1 [9] and type 2 diabetics [10]. Despite the benefits of a regular exercise program, many individuals do not maintain sufficient physical activity and an increasing portion of the adult population engage in no leisure time physical activity [11]. Developing techniques to help motivate individuals to be more physically active can have important public health benefits.

The benefits of exercise are mediated in large part through cardiorespiratory fitness (CRF) [12] and muscular fitness (MF) [13]. Although there is a strong genetic component to CRF it can be improved with regular exercise and physical activity [14]. It is well established that increased CRF is associated with better functional ability, improved cardiovascular health and reduced total mortality [12]. Individual measurements of CRF are a more powerful predictor of mortality than more traditional cardiovascular risk factors such as systolic blood pressure and total cholesterol [15]. CRF is best measured by maximum oxygen uptake $\left(\mathrm{VO}_{2} \mathrm{max}\right)$ representing one's maximum ability to deliver and consume oxygen during activity. Despite the importance and prognostic significance of CRF and $\mathrm{VO}_{2}$ max they are not routinely measured or estimated in the general population or the clinical setting [16]. Measuring $\mathrm{VO}_{2}$ max typically involves a maximal exercise session which limits its availability and utility. A self-paced step test has been validated in a primary care setting as a safe and simple method of approximating $\mathrm{VO}_{2}$ max and CRF [17].

MF and strength also mediate the beneficial effects of exercise. Increased muscular strength is strongly associated with a lower all-cause and CVD mortality [13]. The reduction in all-cause mortality associated with muscular strength has been found to be independent of CRF [18]. Increased muscular strength improves metabolic and cardiovascular risk markers [19] and reduces the risk of developing metabolic syndrome [20] . Exercise, especially resistance training, can increase muscular strength at any age $[21,22]$. Although MF and strength have important metabolic and health implications they also are not routinely assessed in the general population or in the health care setting [23]. A handgrip dynamometer has been shown to be a cost-effective clinical marker of sarcopenia and correlates with lower extremity muscle power and poor mobility [24]. A large longitudinal population study found that measurement of grip strength is a simple, inexpensive risk-stratifying method for all-cause death, cardiovascular death, and CVD [25].

Given the major health implications of CRF and MF we hypothesized that providing individuals with an estimate of their CRF and $\mathrm{VO}_{2}$ max via the step test and MF via grip strength along with educational information and normative data would be effective in motivating an overall increase in subsequent exercise and physical activity. We also secondarily hypothesized that this increase would be through increased cardio and resistance exercise.

\section{Methods}

\section{Study population}

The study was undertaken at the Minnesota State Fair over 12 days in late August and early September 2014 and 2015. Subjects were self-selected when they volunteered to participate while exploring the University of Minnesota's Driven to Discover Building on the Minnesota State Fairgrounds. Exclusion criteria were age $<18$ years, pregnancy, history of heart disease, syncope, chest pain, dyspnea, beta blocker or non-dihydropyridine calcium channel blockers use or evidence of any unstable medical condition. We estimated that we needed to enroll 440 individuals (220 per group) to detect an increase of $15 \%$ in EVS with $95 \%$ confidence level.

\section{Enrollment and testing protocol}

Subjects were randomized in a parallel fashion with 1:1 allocation via sealed envelope to either the control or the intervention group. All subjects provided signed written informed consent and had self-reported weight and height recorded and heart rate and blood pressure measured. The Exercise Vital Sign (EVS) [26] was used to document current exercise activity and was recorded for all subjects. The EVS is the product of the answers to two questions: "On average, how many days per week do you engage in moderate to strenuous exercise like a brisk walk" and "On average how many minutes do you engage in exercise at this level". We also inquired as to the types of self-reported physical activity performed (with examples provided): None, Cardio (walking, running, biking swimming), Sports (tennis, basketball, dancing etc.), Resistance Exercises (weight lifting, resistance 
bands etc.), Lifestyle Activities (yard work, mowing, raking, digging etc.), Balance/Flexibility Exercises (yoga, Tai chi etc.) and Other. We capped the maximum EVS at 840 by limiting reported exercise time to $120 \mathrm{~min} /$ day.

All subjects in both groups were provided educational information on recommended physical activity based on the American College of Sports Medicine Guidance for Prescribing Exercise [27]. Those randomized to the control group did not undergo further testing.

The intervention group had grip strength measured in the dominant arm with a J00105 hand-grip dynamometer (Lafayette Instrument). Subjects were allowed to adjust the dynamometer to their hand size, chose their most comfortable arm position and whether to stand or sit for the testing. The highest value achieved in three attempts was recorded. Subjects were given a brief demonstration of the step test procedure by study staff but no opportunity to practice. Heart rate was recorded pre and post testing and using previously published protocols [15] $\mathrm{VO}_{2}$ max was estimated using the timed 20 step protocol at "normal" speed. No problems or side effects were encountered during any of the measurements. The measured grip strength and the calculated $\mathrm{VO}_{2}$ max were provided to subjects along with age specific norms for both the grip strength [28] and $\mathrm{VO}_{2} \max$ [29]. In 2014 participants were given an age specific "good" norm $\mathrm{VO}_{2} \mathrm{max}$ and in 2015 were provided a "superior" norm for $\mathrm{VO}_{2} \max$ [29] to determine if increasing the normative value would have a greater impact on subsequent exercise behavior.

\section{Follow-up data collection and analysis}

All study participants in both the control and intervention groups were contacted via email at 3, 6 and 12 months following enrollment to determine their current EVS and type of physical activity after which the trial was stopped and no further contacts were attempted. The change in EVS from baseline for both the intervention and control group was compared at 3, 6, and 12 months. Pre-specified subgroup analysis was also performed based on baseline physical activity levels. Participant baseline characteristics were summarized by group using descriptive statistics. Types of exercise were compared between groups using the Chi-square test. For participants who provided data at all 4 time points, the change in EVS over time were evaluated using linear mixed models. Models included fixed effect of intervention, time, and their interaction, and accounted for correlations among repeated measures. Tukey method was used to adjust for multiple comparisons. Subgroup analysis was conducted to examine change in EVS based on baseline physical activity levels. Analysis was performed using Statistical Analysis Software (version 9.3, SAS Institute Inc., Cary, NC).

\section{Results}

Study population and baseline measurement

Total study enrollment was 1315 subjects (776 in 2014 and 539 in 2015) with 659 subjects assigned to the control group and 656 subjects to the intervention group. Mean age was 46.0 (range 18-92), males accounted for $63.0 \%$ of the total, and $92.6 \%$ of all participants were white. Table 1 shows the demographic characteristics and baseline EVS of the control and intervention groups.

The Intervention group's estimated VO2max ranged from 11.2 to $77.3 \mathrm{ml} / \mathrm{kg} / \mathrm{min}$ with a mean of 41.7 (SD 12.1). Maximum grip strength ranged from $10.9-83.2 \mathrm{Kg}$ with a mean of $35.9 \mathrm{Kg}$ (SD 11.9). Nearly all (97.4\%) participants reported some regular exercise activity. The reported types of exercise activity performed at baseline and at follow up is reported in Table 2.

\section{Follow-up EVS}

During the 1 year follow-up responses were received from 823 subjects (62.6\%) with 262 subjects (20\%) responding to all 3 contacts. No additional attempts were made to contact participants if they did not respond to our e-mail. Table 3 shows the follow up EVS values. There was no significant change in the EVS from baseline found in either the control or intervention group $(p=0.99)$.

Among the 262 subjects from both groups who provided data at all 3 follow up intervals 82 had a baseline EVS less than the recommended $150 \mathrm{~min} /$ week. This group, which includes both control and intervention subjects and a baseline EVS $<150$, exhibited an increase in mean EVS over baseline at each follow up point, reaching statistical significance at 6 months (mean EVS $86(\mathrm{SD}=41)$ at baseline vs. mean EVS $146(\mathrm{SD}=135)$ at 6 months, $p<0.05$ ) (Fig. 1). The group meeting or exceeding current recommendations with EVS $>150$ at baseline $(N=180)$ showed no significant change.

\section{Follow-up exercise type}

Although the follow up EVS showed no significant differences in the total amount of physical activity, there were significant differences in the type of exercise activity performed (Table 2). At 3 months and 6 months the intervention group exhibited a significant increase in lifestyle activity $(29.1 \%$ in intervention vs. $20.1 \%$ in control at 3 months, $25.0 \%$ in intervention vs. $18.7 \%$ in control at 6 months, $p<0.05$ for both 3 months and 6 months). The intervention group also significantly increased resistance exercise at 3 months $(42.8 \%$ in intervention vs $31.4 \%$ in control, $p<0.05)$. This increase in resistance exercise was driven by subjects whose baseline grip strength was less than the norm as they exhibited a significant increase in resistance training throughout the 1 year follow up (32\% at baseline vs. $52 \%$ at 3 months, 
Table 1 Baseline demographics

\begin{tabular}{llll}
\hline & Overall & Control & Intervention \\
\hline Number of subjects & 1315 & 659 & 656 \\
Age, mean (range) & $46.0(18-92)$ & $44.9(18-92)$ & $47.1(18-88.5)$ \\
Males, N (\%) & $829(63.0 \%)$ & $418(63.2 \%)$ & $411(62.3 \%)$ \\
Whites, N (\%) & $1218(92.6 \%)$ & $607(92.0 \%)$ & $611(92.6 \%)$ \\
BMl Kg/M ${ }^{2}$, mean (range) & $25.8(16.0-61.2)$ & $26.0(16.0-61.2)$ & $25.6(16.0-56.6)$ \\
EVS minutes/week, mean (SD) & $213.2(176.6)$ & $211.7(168.7)$ & $214.6(184.1)$ \\
\hline
\end{tabular}

$B M$ Body mass index EVS Exercise Vital Sign SD standard deviation

$49 \%$ at 6 months, and $54 \%$ at 12 months, $p<0.05$ ). Subjects with grip strength at or above norm significantly increased resistance training at only 6 months $(36 \%$ at baseline vs. $51 \%$ at 6 months, $p<0.05$ ) (Table 4).

\section{Effect of CRF measurements on subsequent EVS}

Below norm $\mathrm{VO}_{2 \max }(p=1)$ or grip strength $(p=0.75)$ had no effect on subsequent reported EVS as seen in Table 5. Providing a higher normative value for $\mathrm{VO}_{2 \max }$ also had no effect on subsequent reported EVS $(p=$ 0.62).

\section{Discussion}

We did not find that estimates of CRF and MF increased total physical activity over the subsequent year as measured by the EVS. We did find a significant increase in both lifestyle exercise and resistance type exercise in an already very physically active population. Having a below norm grip strength was associated with a significant increase in resistance training throughout the subsequent year. We found that individuals not meeting current recommended physical activity recommendations (EVS $<$ $150 \mathrm{~min} /$ week) showed a significant increase in their EVS at 6 months of follow up. This study indicates that recording EVS, providing exercise recommendations and estimating CRF and MF could provide both a useful incentive to stimulate greater interest in exercise, lifestyle physical activity and resistance training. To our knowledge no other study has investigated the effect on subsequent physical activity of $\mathrm{VO}_{2}$ max estimates or grip strength.

Recognizing the importance of exercise and physical activity to good health, the Surgeon General and others have called for regular assessments of an individual's physical activity [30, 31]. The National Physical Activity Plan asks healthcare systems to prioritize physical activity assessment, advice, and promotion and regularly assess physical activity as a "vital sign" [32]. The EVS has been advocated as a tool to help accomplish this goal [26]. We used the EVS to quantify the exercise activity in our subjects. It is easily calculated with just two questions and corresponds to current exercise guidelines recommending $150 \mathrm{~min}$ of moderately vigorous physical activity per week [27].

Our study population was done in random selfselected volunteers. These volunteers were already very physically active as indicated by the mean EVS of 223.2 with a median value of 180 , significantly exceeding current EVS recommendation of 150 [27]. The high level of pre-existing physical activity likely attenuated the impact of the fitness measurements on their future physical activity and limited the utility of the EVS as a measurement tool [33]. We did find, however, that the subjects not meeting current physical activity guidelines did exhibit a significant increase in their reported EVS at 6 months. Both the control and intervention groups exhibited this increase indicating that recording the EVS and providing information on current exercise recommendations likely influenced this change. This observation

Table 2 Type of exercise reported by subjects at baseline and follow-up

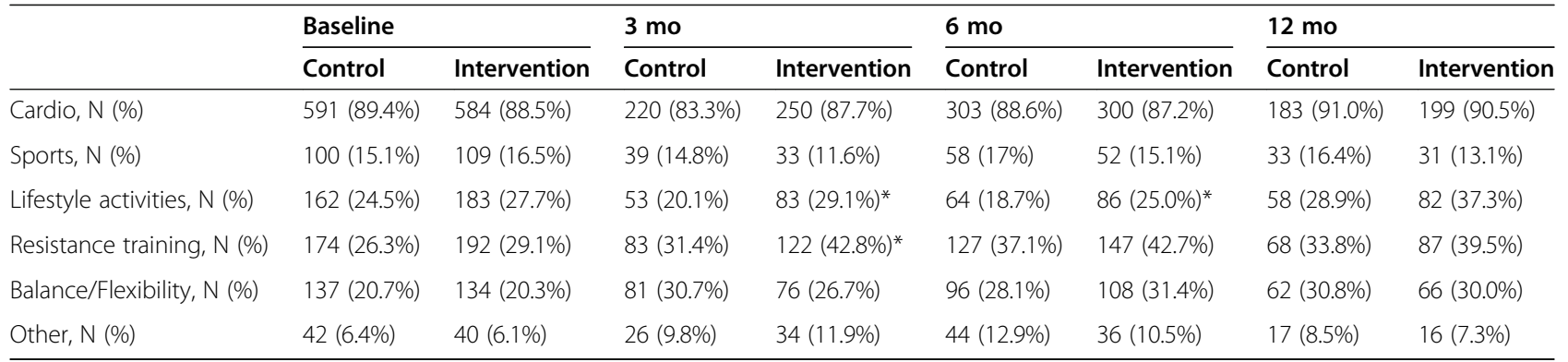

$N$ number *Indicates $p<.05$ for difference in change from baseline between study groups (Intervention vs. Control) 
Table 3 Change in EVS (min/week) over time

\begin{tabular}{lllll}
\hline & Baseline EVS & $\mathbf{3}$ mo EVS & $\mathbf{6}$ mo EVS & $\mathbf{1 2}$ mo EVS \\
\hline Control & & & & \\
Mean (SD) & $212(169)$ & $193(170)$ & $216(166)$ & $221(161)$ \\
$\mathrm{N}$ & 656 & 263 & 341 & 200 \\
Intervention & & & & \\
Mean (SD) & $215(184)$ & $215(173)$ & $219(166)$ & $250(193)$ \\
$\mathrm{N}$ & 659 & 283 & 343 & 219 \\
\hline
\end{tabular}

$N$ number of responses, EVS Exercise Vital Sign

validates calls for recording exercise as a vital sign. It has previously been reported that systematically recording the EVS during outpatient visits was associated with significant changes in exercise-related clinical counseling and documentation [34]. Consistently meeting physical activity guidelines as measured by the EVS is also strongly associated with a reduced risk for severe COVID-19 outcomes among infected adults [35].

In addition to obtaining the EVS we recorded the types of physical activity both initially and in follow up. We found that the intervention group significantly increased their reported resistance training and lifestyle physical activity relative to controls at 3-months followup, despite the much less favorable climate for these activities during winter. The increase in lifestyle activity was sustained at 6 months follow up. A significant increase in resistance training was observed throughout the following year in those individuals with a reported grip strength less than the reported norm. Below norm grip strength appears to stimulate interest in strength training activities in this already very active population. At baseline, $88 \%$ of our study population reported participating in some form of cardiovascular exercise but only $28 \%$ reported participation in resistance type exercise. This lower level of resistance training is consistent with prior surveys showing only $21.9 \%$ of Americans meet muscle strengthening guidelines [34]. This provides greater potential for our assessments and recommendations to have an impact on resistance exercise activity. We did not observe that those subjects with grip strength below the norm increased their EVS even though they did increase their resistance training.

Cardiorespiratory Fitness as measured by $\mathrm{VO}_{2} \max$ is an important indicator of overall health and has significant prognostic implications [12, 14, 15]. Recognizing the significance of CRF the American Heart Association has called for the inclusion of CRF measurement or estimation in routine clinical practice [16]. Despite its importance it is not typically measured in a clinical encounter. This relates to the difficulty of formal $\mathrm{VO}_{2} \max$ measurements. Other forms of estimating $\mathrm{VO}_{2} \max$ such as maximal or sub-maximal treadmill or bicycle exercise testing are also not suited to routine use. $\mathrm{VO}_{2} \max$ can easily be estimated by several formulas based on demographics and reported exercise habits [36, 37]. Estimating CRF from one of these formulas has been associated with CVD and all-cause mortality independent of other risk factors [38]. The estimated CRF from formulas however are significantly influenced by the subjective reporting of exercise activity. We elected to use a step test that had been previously validated in a geriatric population [15]. This test in younger individuals and other populations has been found to be less accurate in the measured $\mathrm{VO}_{2}$ max yet still felt to be useful in classifying CRF [39]. When this step test has been used to measure CRF to aid in exercise prescription a significant improvement in $\mathrm{VO}_{2} \max$ at 12 months was found compared with baseline measures [40].

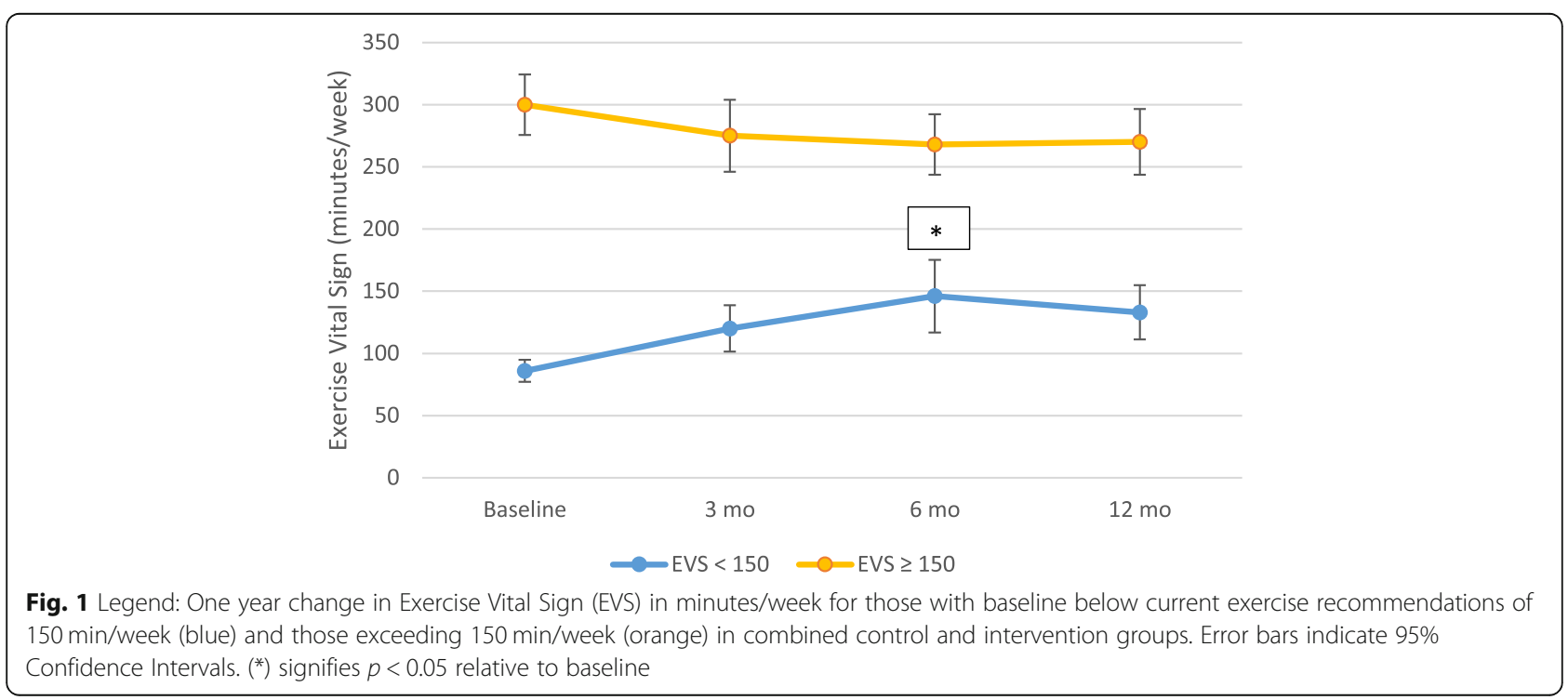


Table 4 Number (\%) of subjects reporting resistance training by baseline grip strength norms

\begin{tabular}{lllll}
\hline & Baseline & $\mathbf{3}$ mo & $\mathbf{6}$ mo & 12 mo \\
\hline Baseline grip strength $<$ norm $(N=69)$ & $22(32 \%)$ & $36^{*}(52 \%)$ & $34^{*}(49 \%)$ & $37^{*}(54 \%)$ \\
Baseline grip strength $\geq$ norm $(N=67)$ & $24(36 \%)$ & $31(46 \%)$ & $34^{*}(51 \%)$ & $28(42 \%)$ \\
\hline
\end{tabular}

*indicates $p<0.05$ for change from baselin

We felt that providing a CRF estimate requiring actual physical activity with the step test would more likely influence future exercise behavior than an estimate of CRF from a formula. We did not observe significant changes in the EVS in either the control group or the intervention group over 1 year of follow up. The fitness assessments did not appear to influence this very active population's physical activity as measured by the EVS. We also did not observe an increase in follow up EVS in those individuals who were reported to have an estimated $\mathrm{VO}_{2}$ max below the provided norm even when the norm was increased from "good" to "superior".

We used a hand-grip dynamometer to estimate muscular strength. This test is inexpensive, convenient and previously demonstrated in multiple studies to be a clinically significant marker of sarcopenia and correlate with lower extremity muscle power and mobility (20). In a large longitudinal population study, measurement of grip strength was found to be a simple, inexpensive riskstratifying method for all-cause death, cardiovascular death, and CVD (21). Grip strength is predictive of mortality in both young adults [41] and middle age [42]. Low grip strength has been documented to correlate with increased disability in the elderly [43], greater risk for hospitalization [44], cognitive decline [45] and nutritional status [46]. We used grip strength to estimate MF and felt that this measurement would contribute to increased physical activity and resistance training. We did observe an increase in resistance training but not total exercise time. The increase in resistance exercise was largely driven by those individuals with grip strength below norm. Given the overall high level of baseline physical activity and lower level of resistance training at baseline it appears that having a below norm grip strength shifted physical activity to resistance training from other activities.
The strengths of this study are its size and diversity. The study participants exhibited a wide range of age (18-92 mean 46) and BMI (16-61, mean 25).

The study limitations were the self-selected population that was predominately white $(92.3 \%)$ and already very active as exhibited by the high baseline EVS. In this active group the EVS may have been less accurate in measuring their physical activity as the EVS has been shown to under-report physical activity measured by accelerometer and may be best used for identifying individuals not meeting current physical activity guidelines [33]. Other limitations of using the EVS are the absence of a specific time frame and the inability to differentiate exercise intensity. This active group also may have been more receptive to feedback on their CRF and MF accounting for the short term significant increases in the lifestyle and resistance physical activity but with less potential to observe an increase in EVS over time. The results are also limited by the self-reported nature of the data and follow up data being provided from only $62.5 \%$ of the study population. We also did not perform follow up CRF or MF measurements.

In summary we did not find that our measurements of CRF and MF using a step test and grip strength increased overall exercise or physical activity as measured by the EVS during the ensuing year. The utility of our intervention was likely limited by a self-selected very active population. Less active individuals in both the control and intervention groups (those not meeting current exercise guidelines) did significantly increase their reported exercise activity at 6 months. We found that the fitness measurements appeared to stimulate an increase in lifestyle and resistance training exercise at short term follow up and that the increase in resistance training was largely driven by those having a below norm grip strength. This indicates a potential benefit of recording the EVS and providing current exercise

Table 5 Follow up EVS relative to normative data

\begin{tabular}{lllll}
\hline & $\begin{array}{l}\text { Baseline EVS } \\
\text { Mean (SD) }\end{array}$ & $\begin{array}{l}\text { 3 mo EVS } \\
\text { Mean (SD) }\end{array}$ & $\begin{array}{l}\mathbf{6} \text { mo EVS } \\
\text { Mean (SD) }\end{array}$ & $\begin{array}{c}12 \text { mo EVS } \\
\text { Mean (SD) }\end{array}$ \\
\hline Baseline VO2max $<$ norm $(N=42)$ & $193(153)$ & $206(175)$ & $221(176)$ & $196(181)$ \\
Baseline VO2max $\geq$ norm $(N=100)$ & $272(194)$ & $244(183)$ & $257(179)$ & $261(191)$ \\
Baseline grip strength $<$ norm $(\mathrm{N}=69)$ & $264(203)$ & $224(175)$ & $246(211)$ & $235(202)$ \\
Baseline grip strength $\geq$ norm $(\mathrm{N}=67)$ & $224(157)$ & $263(202)$ & $252(143)$ & $252(185)$ \\
\hline
\end{tabular}

EVS Exercise Vital Sign (minutes/week), $N$ number of respondents 
recommendations to less active individuals. Even very active individuals may benefit from measuring grip strength and providing norms to stimulate greater participation in resistance training activities. Given these encouraging improvements in exercise activity it may be useful to more widely record EVS and perform CRF and MF estimates.

\section{Conclusions}

In a very active population providing $\mathrm{VO}_{2}$ max estimates and grip strength measurement did not produce an increase in overall physical activity however it did shift activity to increased lifestyle physical activity and resistance training. Recording the EVS and providing exercise recommendations did result in a significant increase in overall physical activity in those individuals not meeting current physical activity recommendations. Wider adoption of the EVS and grip strength measurement could be effective in promoting physical activity and resistance training.

\section{Abbreviations}

CVD: Cardiovascular disease; CRF: Cardiorespiratory fitness;

$\mathrm{VO}_{2}$ max: Maximum oxygen uptake; MF: Muscular fitness; EVS: Exercise vital sign

\section{Acknowledgements \\ None. \\ The study adheres to CONSORT guidelines and a completed CONSORT checklist has been submitted separately.}

\section{Authors' contributions}

JTL, NS and APJO contributed equally to data collection and manuscript draft, JTL manuscript submission, QW statistical analysis. All authors have read and approved the manuscript.

\section{Funding}

Not applicable.

\section{Availability of data and materials}

The datasets used and analyzed during the current study are available from the corresponding author on reasonable request.

\section{Declarations}

\section{Ethics approval and consent to participate}

This study and the consent form were approved by the University of Minnesota Institutional Review Board. Signed written informed consent was obtained from all subjects.

\section{Consent for publication \\ Not applicable.}

\section{Competing interests}

Not applicable.

\section{Author details}

${ }^{1}$ Department of Medicine, Division of General Internal Medicine, University of Minnesota, 420 Delaware St SE, MMC 784, Minneapolis, MN 55455, USA ${ }^{2}$ Department of Medicine, Division of Cardiology, University of Minnesota, Minneapolis, MN, USA. ${ }^{3}$ Clinical/Translational Science Institute, University of Minnesota, Minneapolis, MN, USA.
Received: 27 December 2020 Accepted: 2 June 2021

Published online: 15 June 2021

\section{References}

1. Arem H, Moore SC, Patel A, Hartge P, Berrington de Gonzalez A, Visvanathan $\mathrm{K}$, et al. Leisure time physical activity and mortality. JAMA Intern Med. 2015;175(6):959-67. https://doi.org/10.1001/jamainternmed.201 5.0533.

2. Wen CP, Wai JPM, Tsai MK, Chen CH. Minimal amount of exercise to prolong life: to walk, to run, or just mix it up? J Am Coll Cardiol. 2014;64(5): 482-4. https://doi.org/10.1016/j.jacc.2014.05.026.

3. Shiroma EJ, Lee I-M. Physical activity and cardiovascular health: lessons learned from epidemiological studies across age, gender, and race/ethnicity. Circulation. 2010;122(7):743-52. https://doi.org/10.1161/CIRCULATIONAHA.1 09.914721.

4. Crump C, Sundquist J, Winkleby MA, Sundquist K. Interactive effects of physical fitness and body mass index on the risk of hypertension. JAMA Intern Med. 2016;176(2):210-6. https://doi.org/10.1001/jamainternmed.2015. 7444 .

5. Illiam W, Raus EK, Oseph J, et al. Effects of the amount and intensity of exercise on plasma lipoproteins. N Engl J Med. 2002;347:1483-92.

6. Cooney GM, Dwan K, Greig CA et al. Exercise for depression ( Review ). Cochrane Database Syst Rev 2013, Issue 9. Art. No.: CD004366.

7. Goodwin RD. Association between physical activity and mental disorders among adults in the United States. Prev Med (Baltim). 2003;36:698-703.

8. Zhu W, Wadley VG, Howard VJ, et al. Objectively measured physical activity and cognitive function in older adults. Med Sci Sports Exerc. 2017:49(1):4753. https://doi.org/10.1249/MSS.0000000000001079.

9. Adamo M, Codella R, Casiraghi F, Ferrulli A, Macrì C, Bazzigaluppi E, et al. Active subjects with autoimmune type 1 diabetes have better metabolic profiles than sedentary controls. Cell Transplant. 2017 Jan 24;26(1):23-32. https://doi.org/10.3727/096368916X693022.

10. Codella R, lalacqua M, Terruzzi I, Luzi L. May the force be with you: why resistance training is essential for subjects with type 2 diabetes mellitus without complications. Endocrine. 2018;62(1):14-25. https://doi.org/10.1007/ s12020-018-1603-7.

11. Ladabaum U, Mannalithara A, Myer PA, Singh G. Obesity, abdominal obesity, physical activity, and caloric intake in US adults: 1988 to 2010. Am J Med. 2014;127(8):717-27. https://doi.org/10.1016/j.amjmed.2014.02.026.

12. Kaminsky LA, Arena R, Beckie TM, Brubaker PH, Church TS, Forman DE, et al. The importance of cardiorespiratory fitness in the United States: the need for a national registry: a policy statement from the American Heart Association. Circulation. 2013;127(5):652-62. https://doi.org/10.1161/CIR. 0b013e31827ee100.

13. Kamiya K, Masuda T, Tanaka S, Hamazaki N, Matsue Y, Mezzani A, et al. Quadriceps strength as a predictor of mortality in coronary artery disease. Am J Med. 2015;128(11):1212-9. https://doi.org/10.1016/j.a mimed.2015.06.035.

14. Lavie CJ, Arena R, Swift DL, Johannsen NM, Sui X, Lee DC, et al. Exercise and the cardiovascular system: clinical science and cardiovascular outcomes. Circ Res. 2015;117(2):207-19. https://doi.org/10.1161/CIRCRESAHA.117.305205.

15. Gupta S, Rohatgi A, Ayers CR, Willis BL, Haskell WL, Khera A, et al. Cardiorespiratory fitness and classification of risk of cardiovascular disease mortality. Circulation. 2011;123(13):1377-83. https://doi.org/10.1161/CIRCULA TIONAHA. 110.003236.

16. Ross R, Blair SN, Arena R, Church TS, et al. Importance of assessing cardiorespiratory fitness in clinical practice: a case for fitness as a clinical vital sign: a scientific statement from the american heart association. Circulation, 2016:134(24):e653-99.

17. Petrella RJ, Koval JJ, Cunningham DA, Paterson DH. A self-paced step test to predict aerobic fitness in older adults in the primary care clinic. J Am Geriatr Soc. 2001;49(5):632-8. https://doi.org/10.1046/j.1532-5415.2001.49124.x.

18. Ruiz JR, Sui X, Lobelo F, Morrow JR, Jackson AW, Sjostrom M, et al. Association between muscular strength and mortality in men: prospective cohort study. BMJ. 2008;337(jul01 2):a439. https://doi.org/10.1136/bmj.a439.

19. Roberts CK, Lee MM, Katiraie M, et al. Strength fitness and body weight status on markers of cardiometabolic health. Med Sci Sports Exerc. 2015; 47(6):1211-8. https://doi.org/10.1249/MSS.0000000000000526.

20. Sénéchal M, McGavock JM, Church TS, et al. Cut points of muscle strength associated with metabolic syndrome in men. Med Sci Sports Exerc. 2014; 46(8):1475-81. https://doi.org/10.1249/MSS.0000000000000266. 
21. Harber MP, Konopka AR, Undem MK, Hinkley JM, Minchev K, Kaminsky LA, et al. Aerobic exercise training induces skeletal muscle hypertrophy and age-dependent adaptations in myofiber function in young and older men. J Appl Physiol. 2012;113(9):1495-504. https://doi.org/10.1152/japplphysiol. 00786.2012 .

22. Peterson MD, Rhea MR, Sen A, Gordon PM. Resistance exercise for muscular strength in older adults: a meta-analysis. Ageing Res Rev. 2010;9:226-37.

23. Ibrahim K, May C, Patel HP, Baxter M, Sayer AA, Roberts H. A feasibility study of implementing grip strength measurement into routine hospital practice (GRImP): study protocol. Pilot Feasibility Stud. 2016;2(1):27. https://doi.org/1 0.1186/s40814-016-0067-x.

24. Lauretani F, Russo CR, Bandinelli S, Bartali B, Cavazzini C, di lorio A, et al. Age-associated changes in skeletal muscles and their effect on mobility: an operational diagnosis of sarcopenia. J Appl Physiol. 2003;95(5):1851-60. https://doi.org/10.1152/japplphysiol.00246.2003.

25. Leong DP, Teo KK, Rangarajan S, Lopez-Jaramillo P, Avezum A Jr, Orlandin $A$, et al. Prognostic value of grip strength: findings from the prospective urban rural epidemiology (PURE) study. Lancet. 2015;386(9990):266-73. https://doi.org/10.1016/S0140-6736(14)62000-6.

26. Coleman KJ, Ngor E, Reynolds K, et al. Initial validation of an exercise "vital sign" in electronic medical records. Med Sci Sports Exerc. 2012;44(11):20716. https://doi.org/10.1249/MSS.0b013e3182630ec1.

27. Garber CE, Blissmer B, Deschenes MR, Franklin BA, Lamonte MJ, Lee IM, et al. Quantity and quality of exercise for developing and maintaining cardiorespiratory, musculoskeletal, and neuromotor fitness in apparently healthy adults: guidance for prescribing exercise. Med Sci Sports Exerc. 2011;43(7):1334-59. https://doi.org/10.1249/MSS.0b013e318213fefb.

28. Mathiowetz V, Kashman N, Volland G, Weber K, Dowe M, Rogers S. Grip and pinch strength: normative data for adults. Arch Phys Med Rehabil. 1985; 66(2):69-74.

29. The Physical Fitness Specialist Certification Manual, The Cooper Institute for Aerobics Research, Dallas TX, revised 1997 printed in Advance Fitness Assessment \& Exercise Prescription, 3rd Edition, Vivian H. Heyward, 1998.p48.

30. Office of the Surgeon General (US). Step It Up!: The Surgeon General's Call to Action to Promote Walking and Walkable Communities. Washington (DC): US Department of Health and Human Services; 2015.

31. Foster C, Hillsdon M, Thorogood M, Kaur A. Interventions for promoting physical activity. Cochrane Database Syst Rev. 2014;9:1-90.

32. National Physical Activity Plan Alliance (2016). U.S. National Physical Activity Plan. Available at: http://physicalactivityplan.org/docs/2016NPAP_Fina Iforwebsite.pdf

33. Joseph RP, Keller C, Adams MA, Ainsworth BE. Validity of two brief physical activity questionnaires with accelerometers among African-American women. Prim Health Care Res Dev. 2016;17(3):265-76. https://doi.org/10.101 7/S1463423615000390.

34. Carlson SA, Fulton JE, Schoenborn CA, Loustalot F. Trend and prevalence estimates based on the 2008 physical activity guidelines for Americans. Am J Prev Med. 2010;39:305-13.

35. Sallis R, Young DR, Tartof SY, et al. Physical inactivity is associated with a higher risk for severe COVID-19 outcomes: a study in 48,440 adult patients. Br J Sports Med Published Online First: 13 April 2021. doi: https://doi.org/1 0.1136/bjsports-2021-104080

36. Nes BM, Janszky I, Vatten LJ, Nilsen TIL, Aspenes ST, WislØff U. Estimating VO2peak from a nonexercise prediction model: the HUNT study, Norway. Med Sci Sports Exerc. 2011;43(11):2024-30. https://doi.org/10.1249/MSS. Ob013e31821d3f6f

37. O'Donovan G, Bakrania K, Ghouri N, et al. Nonexercise equations to estimate fitness in White European and south Asian men. Med Sci Sports Exerc. 2016; 48(5):854-9. https://doi.org/10.1249/MSS.0000000000000836.

38. Nauman J, Nes BM, Lavie CJ, et al. Prediction of cardiovascular mortality by estimated cardiorespiratory fitness independent of traditional risk factors: the HUNT study. Mayo Clin Proc. 2016;92:1-10.

39. Vidoni ED, Mattlage A, Mahnken J, Burns JM, McDonough J, Billinger SA. Validity of the step test for exercise prescription: no extension to a larger age range. J Aging Phys Act. 2013;21(4):444-54. https://doi.org/10.1123/ja pa.21.4.444.

40. Petrella RJ, Lattanzio CN, Shapiro S, Overend T. Improving aerobic fitness in older adults: effects of a physician-based exercise counseling and prescription program. Can Fam Physician. 2010;56(5):e191-200.
41. Ortega FB, Silventoinen K, Tynelius P, Rasmussen F. Muscular strength in male adolescents and premature death: cohort study of one million participants. BMJ. 2012;345(nov20 3):e7279. https://doi.org/10.1136/bmj.e7279.

42. Rantanen T, Masaki K, He Q, Ross GW, Willcox BJ, White L. Midlife muscle strength and human longevity up to age 100 years: a 44-year prospective study among a decedent cohort. Age (Omaha). 2012;34(3):563-70. https:// doi.org/10.1007/s11357-011-9256-y.

43. Giampaoli S, Ferrucci L, Cecchi F, Lo Noce C, Poce A, Dima F, et al. Handgrip strength predicts incident disability in non-disabled older men. Age Ageing. 1999;28(3):283-8. https://doi.org/10.1093/ageing/28.3.283.

44. Cawthon PM, Fox KM, Gandra SR, Delmonico MJ, Chiou CF, Anthony MS, et al. Do muscle mass, muscle density, strength, and physical function similarly influence risk of hospitalization in older adults? J Am Geriatr Soc. 2009;57(8):1411-9. https://doi.org/10.1111/j.1532-5415.2009.02366.x.

45. Auyeung TW, Lee JSW, Kwok T, Woo J. Physical frailty predicts future cognitive decline - a four-year prospective study in 2737 cognitively normal older adults. J Nutr Heal Aging. 2011;15(8):690-4. https://doi.org/10.1007/s12 603-011-0110-9.

46. Norman K, Stobäus N, Gonzalez MC, Schulzke JD, Pirlich M. Hand grip strength: outcome predictor and marker of nutritional status. Clin Nutr. 2011;30(2):135-42. https://doi.org/10.1016/j.clnu.2010.09.010.

\section{Publisher's Note}

Springer Nature remains neutral with regard to jurisdictional claims in published maps and institutional affiliations.
Ready to submit your research? Choose BMC and benefit from:

- fast, convenient online submission

- thorough peer review by experienced researchers in your field

- rapid publication on acceptance

- support for research data, including large and complex data types

- gold Open Access which fosters wider collaboration and increased citations

- maximum visibility for your research: over $100 \mathrm{M}$ website views per year

At BMC, research is always in progress.

Learn more biomedcentral.com/submissions 